FROM THE GROUNDS UP 
This page intentionally left blank 


\title{
From the Grounds Up
}

\author{
BUILDING AN EXPORT ECONOMY \\ IN SOUTHERN MEXICO
}

Casey Marina Lurtz

STANFORD UNIVERSITY PRESS

STANFORD, CALIFORNIA 
STANFORD UNIVERSITY PRESS

Stanford, California

(C) 2019 by the Board of Trustees of the Leland Stanford Junior University. All rights reserved.

No part of this book may be reproduced or transmitted in any form or by any means, electronic or mechanical, including photocopying and recording, or in any information storage or retrieval system without the prior written permission of Stanford University Press.

Printed in the United States of America on acid-free, archival-quality paper.

Library of Congress Cataloging-in-Publication Data

Names: Lurtz, Casey Marina, author.

Title: From the grounds up : building an export economy in southern Mexico / Casey Marina Lurtz.

Description: Stanford, California : Stanford University Press, 2019. I Includes bibliographical references and index.

Identifiers: LCCN 2018033670 | ISBN 978I503603899 (cloth : alk. paper) | ISBN 978 I 503608474 (electronic)

Subjects: LCSH: Coffee industry-Mexico-Soconusco (Region)History-2oth century. I Agricultural industries-Mexico-Soconusco (Region)—History—2oth century. I Soconusco (Mexico : Region)— Commerce-History—2oth century. I Soconusco (Mexico : Region)Economic conditions-2oth century.

Classification: LCC HD9I99.M63 .L87 20I9 I DDC 382/.6097275-dc23

LC record available at https://lccn.loc.gov/201 8033670

Typeset by Kevin Barrett Kane in Io/I2.5 Sabon

Cover design: Rob Ehle

Cover photo: Cuilco River, Finca La Chiripa, Soconusco, Mexico, I930. Courtesy Jose Toriello, Finca Hamburgo. 